\title{
openheart Troponin: a risk marker beyond heart disease
}

\author{
Rodney De Palma, ${ }^{1}$ Pascal Meier ${ }^{1,2}$
}

To cite: De Palma R, Meier $P$. Troponin: a risk marker beyond heart disease. Open Heart 2014;1:e000009. doi:10.1136/openhrt-2013000009

Accepted 17 January 2014

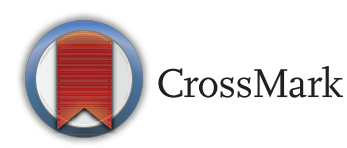

\section{SLinked}

- http://dx.doi.org/10.1136/ openhrt-2013-000001

- http://dx.doi.org/10.1136/ openhrt-2013-000002

\section{${ }^{1}$ The Heart Hospital,} University College London Hospitals UCLH, London, UK ${ }^{2}$ Yale Medical School, New Haven, Connecticut, USA

Correspondence to Dr Rodney de Palma; rodney_de_palma@icloud. com
The advent of cardiac troponin assays has revolutionised the daily assessment and management of patients presenting to emergency rooms with undifferentiated chest symptoms suspicious for acute cardiac ischaemia. This has enhanced the identification of myocyte necrosis thus forming a key part of the contemporary definition for myocardial infarction (MI). ${ }^{1}$

Seminal work by Katus et $a t^{2}$ led to the demonstration by Hamm et $a \hat{l}$ of the clinical applicability of the test in acute coronary syndromes (ACS). Negative troponin being associated with a good prognosis and a positive troponin with an increased frequency of adverse cardiovascular events. Subsequent technical refinements have produced four generations of assays in order to attain the guideline standard of $<10 \%$ coefficient of variation at the 99th centile of a normal reference population. Point-of-care systems have also been developed and deployed by some to reduce the turnaround time of results and improve patient's flow in busy departments.

The relationship between troponin detection in these assays and poor prognosis has been seen not only in ACS but also in myocardial disease, following cardiac procedures, non-cardiac procedures, acute neurological disease, acute respiratory disease chronic renal disease, pulmonary embolism, exposure to certain toxins, sepsis and critical illness of any aetiology. Indeed, the prognosis of troponin-positive non-ACS is generally worse than in troponin-positive ACS.

Recently high-sensitivity cardiac troponin (hs-cTn) assays have become commercially available. These assays mark a seismic shift in the ability to detect troponin at much lower concentrations such that for the majority of the normal reference population there will be a detectable level. While the increased sensitivity of these tests for diagnosing disease above threshold 99th centile values has been a welcome advance, particularly for emergency care practitioners, the loss in specificity has posed challenges to other physicians trying to determine the diagnosis or primary source of disease process as well as the acuity of disease. The use of assay-specific absolute or percentage changes in troponin level has been utilised to help navigate these challenges by identifying acute injury. ${ }^{4}$ Nevertheless, the distinction between type 1 MI (spontaneous 'wild-type' MI), type 2 MI (secondary to ischaemia related to supply/ demand imbalance rather than plaque rupture or coronary occlusion) and myocardial injury has been made much more taxing.

The paper of Hosieth et $a \bar{l}$ in this issue adds novel data to the area with their singlecentre evaluation of hs-cTn dynamic change compared with a stable pattern in unselected acute exacerbations of chronic obstructive pulmonary disease (AECOPD). ${ }^{6}$ Despite the inherent limitations of observational studies and the small size of their cohort, the findings are provocative. Their striking observation was that a stable elevated hs-Tn was associated with a worse survival at a median follow-up of 1.8 years compared with those with dynamic change. The stable pattern median was $38.2 \mathrm{pg} / \mathrm{mL}$. Also notable was that $78 \%$ $(65 / 83)$ of the AECOPD cohort had cTn greater than the 99 th centile $(0.014 \mathrm{pg} / \mathrm{mL}$ for high-sensitivity troponin $\mathrm{T}$ ) with over one-third $(23 / 65)$ satisfying criteria for type $2 \mathrm{MI}$.

What can one make from these findings? The study adds to previous work showing a raised troponin in AECOPD is strongly associated with mortality. ${ }^{7} 8$ The use of hs-cTn without reference to timing of acute symptoms and lack of standardised sample draws may be problematic due to potential misclassification between the two categories evaluated. A recent study, however, has described similar findings in non-ST segment elevation MI. In that work, the authors speculated that as hs-cTn assays reach plateau levels more rapidly than fourth-generation conventional platforms, 'stable' elevated values may reflect a 'late presenting' acute patient rather than a chronic process accounting for a worse prognosis than those with dynamic change. ${ }^{9}$ 
These data also pose broader questions regarding the genesis of troponin detected and therapeutic implications. Release of troponin is considered to reflect ischaemic myocardial necrosis. This, however, may be an oversimplification. Other mechanisms may also be clinically important such as apoptosis, integrin receptor-linked release, membrane permeability, myocyte stretch and membrane blebbing. ${ }^{10}$ Further research is needed to elucidate the role of these putative mechanisms. The guideline committee for the definition of MI may in time use data pertinent to this pathophysiology to further clarify their work.

Finally how to modify management in cases of elevated troponin (stable or dynamic) in non-ACS groups remains to be fully understood. The high-ST-segment elevation ACS study presented recently highlights this issue. In a prospective cohort of undifferentiated suspected ACS for each spontaneous MI (type 1), there were three type $2 \mathrm{MIs}$, and nearly one-third of which were respiratory-related diseases. Increasing the use of cardiac investigations and therapies, however, in type 2 MI did not alter hard outcomes. ${ }^{11}$

Elevated troponin for the clinician is a signal of poor prognosis for acutely ill patient groups including AECOPD. The pattern of hs-Tn release appears to be important and suggests that a binary approach to determine whether troponin is detectable may no longer be sufficient. For AECOPD, the link between troponin, pattern of release, mortality and therapeutic implications remains elusive.

This paper of Hosieth et al illustrates, similarly to a recent publication in this journal by Andell $e t a l,{ }^{12}$ the possible interaction between lung and heart disease. There seems to be a relevant link between chronic obstructive lung disease (COPD) and myocardial necrosis with regard to clinical outcomes. Patients with acute exacerbation of COPD experiencing myocardial necrosis (raised troponins) seem to have a worse prognosis. Similarly, patients presenting with an acute MI and underlying COPD have a significantly worse outcome. As also highlighted in the accompanying editorial, Shashank and Gurm, there seems to be a 'double jeopardy of chronic obstructive pulmonary disease and ischaemic heart disease', ${ }^{13}$ which we need to become more aware of.
Contributors RDP drafted the paper, PM revised it critically for its intellectual content.

\section{Competing interests None.}

Provenance and peer review Commissioned; internally peer reviewed.

Open Access This is an Open Access article distributed in accordance with the Creative Commons Attribution Non Commercial (CC BY-NC 3.0) license, which permits others to distribute, remix, adapt, build upon this work noncommercially, and license their derivative works on different terms, provided the original work is properly cited and the use is non-commercial. See: http:// creativecommons.org/licenses/by-nc/3.0/

\section{REFERENCES}

1. Thygesen K, Alpert JS, Jaffe AS, et al. Third universal definition of myocardial infarction. Circulation 2012;126:2020-35.

2. Katus HA, Looser S, Hallermayer K, et al. Development and in vitro characterization of a new immunoassay of cardiac troponin T. Clin Chem 1992;38:386-93.

3. Hamm CW, Goldmann BU, Heeschen C, et al. Emergency room triage of patients with acute chest pain by means of rapid testing for cardiac troponin T or troponin I. N Engl J Med 1997;337:1648-53.

4. Thygesen K, Mair J, Giannitsis E, et al. How to use high-sensitivity cardiac troponins in acute cardiac care. Eur Heart $J$ 2012;33:2252-7.

5. Høiseth AD, Neukamm A, Hagve T-A, et al. The clinical value of serial measurement of high-sensitivity cardiac troponin $\mathrm{T}$ in acute exacerbations of chronic obstructive pulmonary disease. Open Heart 2014;1:e000001. doi:10.1136/openhrt-2013-000001

6. Bjurman $\mathrm{C}$, Larsson $\mathrm{M}$, Johanson $\mathrm{P}$, et al. Small changes in toponin T levels are common in patients with non-ST-segment elevation myocardial infarction and are linked to higher mortality. J Am Coll Cardiol 2013;62:1231-8.

7. Soyseth $\mathrm{V}$, Bhatnagar $\mathrm{R}$, Holmedahl $\mathrm{NH}$, et al. Acute exacerbation of COPD is associated with fourfold elevation of cardiac troponin $\mathrm{T}$. Heart 2013;99:122-6.

8. Høiseth AD, Neukamm A, Karlsson BD, et al. Elevated high-sensitivity cardiac troponin $\mathrm{T}$ is associated with increased mortality after acute exacerbation of chronic obstructive pulmonary disease. Thorax 2011;66:775-81.

9. Mueller M, Biener M, Vafaie M. Absolute and relative kinetic changes of high-sensitivity cardiac troponin $\mathrm{T}$ in acute coronary syndrome and in patients with increased troponin in the absence of acute coronary syndrome. Clin Chem 2012;58:209-18.

10. White HD. Pathobiology of troponin elevations: do elevations occu with myocardial ischemia as well as necrosis? J Am Coll Cardiol 2011;57:2406-8.

11. Mills N. Clinical Trial Update Hot Line III: updates on risk and outcome. Presented at: the European Society of Cardiology Congress; 31 August-4 September. Amsterdam, 2013.

12. Andell $P$, Koul $S$, Martinsson A, et al. Impact of chronic obstructive pulmonary disease on morbidity and mortality after myocardial infarction. Open Heart 2014;1:e000002. doi:10.1136/openhrt-2013000002

13. Sinha SS, Gurm SH. The double jeopardy of chronic obstructive pulmonary disease and myocardial Infarction. Open Heart 2014;1: e000010. doi:10.1136/openhrt-2013-000010 